\title{
Hesitancy Toward a COVID-19 Vaccine
}

\author{
Linda Thunström, ${ }^{1}$ Madison Ashworth, ${ }^{1}$ David Finnoff, ${ }^{1}$ and Stephen C. Newbold ${ }^{1}$
}

Department of Economics, University of Wyoming, Laramie, WY 82071

\begin{abstract}
The scientific community has come together in a mass mobilization to combat the public health risks of COVID-19, including efforts to develop a vaccine. However, the success of any vaccine depends on the share of the population that gets vaccinated. We designed a survey experiment in which a nationally representative sample of 3,133 adults in the USA stated their intentions to vaccinate themselves and their children for COVID-19. The factors that we varied across treatments were: the stated severity and infectiousness of COVID-19 and the stated source of the risk information (White House or the Centers for Disease Control). We find that $20 \%$ of people in the USA intend to decline the vaccine. We find no statistically significant effect on vaccine intentions from the severity of COVID-19. In contrast, we find that the degree of infectiousness of the coronavirus influences vaccine intentions and that inconsistent risk messages from public health experts and elected officials may reduce vaccine uptake. However, the most important determinants of COVID-19 vaccine hesitancy seem to be distrust of the vaccine safety (including uncertainty due to vaccine novelty), as well as general vaccine avoidance, as implied by not having had a flu shot in the last two years.
\end{abstract}

\section{INTRODUCTION}

Vaccines have historically proven to be highly successful and cost-effective tools for disease prevention in humans (Rémy et al., 2015), domesticated species (Roth, 2011), and expansion of their use in wild species has been advocated (Cross et al., 2007). In the face of the current pandemic, scientists from all over the world have come together to rapidly develop a vaccine for COVID-19 (Callaway, 2020). By April 2020, more than 100 COVID-19 vaccine candi-

Supplementary Information: The online version contains supplementary material available at https://doi.org/10.1007/s10393-021-01524-0.

Published online: June 4, 2021

Correspondence to: Linda Thunström, e-mail: lthunstr@uwyo.edu dates had been developed, several of which quickly advanced to being tested on humans (Le et al., 2020).

However, the effectiveness of a COVID-19 vaccine in controlling the spread of disease depends on the coverage, or uptake level, of the vaccine across a population. A sufficiently high uptake of an effective vaccine may generate herd immunity (a scenario where most people are immune to the virus, preventing it from spreading in the population; e.g., Fine et al., 2011), which protects also those who are still susceptible to the virus. ${ }^{1} \mathrm{~A}$ barrier to reaching herd

\footnotetext{
${ }^{1}$ The threshold for herd immunity in the population (i.e., the proportion of the population that needs to be immune, either from a vaccine, previous infections or both, to ensure herd immunity) is typically inferred from the basic reproduction number for COVID-19, R0. R0 estimates vary across multiple dimensions, such as data availability, geographical location and methods used to produce the estimates (Liu et al., 2020). In the beginning of the pandemic, Sanche et al. (2020) used data
} 
immunity, or a vaccine that protects a large share of the population, is, however, the prevalence of people who refuse or are hesitant to take vaccines (MacDonald, 2015). This share of the US population has grown in recent years (Dubé et al., 2013; Olive et al., 2018). For instance, the uptake level of seasonal influenza vaccines has declined, in part due to vaccine hesitancy (Larson, 2018). During the season 2017-2018, only $37 \%$ of adults got the flu vaccine, even though that flu season was particularly severe (CDC, 2020a). Recent measles outbreaks in the USA and elsewhere illustrate the importance of vaccine hesitancy to public health, as the vaccine had succeeded in extinguishing measles in the USA, but under and non-vaccinated communities contributed to its reappearance (De Serres et al., 2013; Sarkar et al., 2019). The World Health Organization (WHO) named vaccine hesitancy one of the top 10 threats to global health in 2019 (WHO, 2020a).

We examine the prevalence and determinants of avoidance of a COVID-19 vaccine in the USA, early in the pandemic-our data collection was conducted in the end of March 2020. We also examine how vaccine avoidance is affected by information about health risks associated with SARS-CoV-2 (the novel coronavirus), including conflicting risk messages from public authorities. Risk perceptions have been shown to be key to vaccine decisions (Brewer et al., 2007), and effective risk communication is acknowledged as the pillar of a coordinated response to infectious disease outbreaks (Sell, 2017). Observational data suggest that risk information provided in the beginning of the COVID-19 outbreak in the USA affected people's health behavior in response to the pandemic (Bursztyn et al., 2020; Simonov et al., 2020). We examine whether inconsistent risk messages may similarly affect health behavior-in our case willingness to vaccinate. Previous studies suggest beliefs about health risk are affected by who communicates the risk message (e.g., Frewer et al., 1996; Breakwell, 2000; Calman and Curtis, 2010). We focus on inconsistent risk messages delivered by public health ex-

from outbreaks in China and estimated R0 to be as high as 5.7, implying herd immunity may be reached first when $82.5 \%$ of the population is immune (Keeling and Rohani, 2008). Most estimates, across countries and regions, range between 2.5 and 4 (Fontanet and Gauchemez, 2020). Based on that range, the classical formula for herd immunity (1-1/R0) generates a herd immunity threshold within 60 to 75 percent of the population being immune. However, local variations can be large. Sy et al. (2020) estimated R0 for COVID-19 at the county level within the U.S. and found estimates ranging from 0.4 to 12.4 , with a median county level R0 of 1.66. Further, the classic formula for herd immunity may be too simplistic to generate a good approximation of the herd immunity threshold (Aguas et al., 2020). perts (i.e., the CDC) and White House officials, given these authorities regularly address the pandemic in the US media.

To measure vaccine avoidance and how it depends on risk levels and risk communication, we designed a survey experiment that elicited vaccination intentions of adults and their children. Participants consisted of a nationally representative sample $(N=3133)$ of US adults. They were randomized into eight treatment groups across which we varied information about the probability of infection, the conditional mortality ratio from COVID-19 (i.e., the infection fatality ratio, IFR), and whether the different health authorities in the USA provide consistent risk information. Specifically, we examined how vaccine avoidance is affected by elected or appointed White House officials communicating lower risks from COVID-19 than public health experts at the Centers for Disease Control (CDC). The lower level of risk communicated by the White House, compared to that of the CDC, is in line with reporting in popular media at the onset of the current pandemic (e.g., CNN, 2020; MSN, 2020). ${ }^{2}$

While vaccine hesitancy is growing, hesitancy is not equivalent to refusal-many people who are vaccine hesitant do not entirely refuse vaccines. Instead, they either delay vaccines or are willing to take some vaccines but not others (Dubé et al., 2013). Also relevant for a COVID-19 vaccine is the observation that people are more likely to reject new vaccines than familiar ones (Dubé et al., 2013). A US-wide study found that around $10 \%$ of the population refuse all vaccines, including seasonal influenza vaccines and those that comprise the recommended vaccine schedule for children, while around $5 \%$ refuse only one vaccine. A substantial share $(40 \%)$ of those who agreed to at least one vaccine still expressed concerns about vaccines (ASTHO, 2010). The fact that many who are vaccine hesitant are likely to take some vaccines, while perhaps delayed, means it is possible that a portion of those currently reluctant to vaccinate can be swayed (Leask, 2011). Here, risk communication may play an important role, given the correlation between perceived risks and vaccine acceptance (Brewer et al., 2007).

\footnotetext{
${ }^{2}$ Our study relates to a rich body of literature on consumer responses to conflicting information, spanning multiple scientific disciplines. While not an exhaustive list, examples of important work in this area are Viscusi and Magat (1992), Magat and Viscusi (1992), Viscusi (1997), Viscusi et al. (1999), Rodgers (1999), Fox et al. (2002), Hoehn and Randall (2002), Cameron (2005), Rousu and Shogren (2006), Kelly et al. (2012), Carpenter et al. (2014) Hämeen-Anttila et al. (2014), Pushkarskaya et al. (2015), and Binder et al. (2016).
} 
Our study provides important knowledge at a critical point in time. Measuring the share of the population that is reluctant to be vaccinated for COVID-19 can help policy makers, healthcare workers, and other authorities to plan ahead toward minimizing the impact on public health from vaccine hesitancy. This might involve tailored public communication programs designed to persuade vaccinehesitant individuals to accept a COVID-19 vaccine, or increased efforts to ensure a high vaccine uptake level among the remainder of the population, or both. Knowing why people are hesitant to accept a COVID-19 vaccine may enable design of more effective efforts to increase the overall level of vaccine uptake in the general population.

The remainder of this paper is structured as follows. Section 2 describes the survey experiment, Sect. 3 presents the results of the experiment and Sect. 4 concludes by discussing the results, limitations of the current study, and avenues for future research on avoidance of a COVID-19 vaccine.

\section{Survey to Examine Vaccine Intentions AND THEIR DETERMINANTS}

To examine people's willingness to vaccinate for COVID-19, we designed a survey experiment in which participants were asked whether they would choose to vaccinate themselves or their children. The survey experiment was approved by the IRB at University of Wyoming and was pre-registered in the AEA RCT registry (RCT ID: AEARCTR-0005576).

We used a between-subjects experimental design with eight information treatments $(2 \times 2 \times 2)$. The experiment varies information on (1) the probability of the average American catching the coronavirus, (2) the IFR, i.e., the probability of the average American dying if infected, and (3) the source of information for the probability of catching COVID-19 (CDC only/CDC jointly with the White House).

While there are clear benefits to measuring the prevalence and implications of vaccine avoidance before a vaccine is available, doing so comes with methodological challenges-at the time the survey was administrated (the end of March 2020), the true probability of infection in the USA and the mortality rate from COVID-19 were still highly uncertain, partly due to limited testing. ${ }^{3}$ For this

\footnotetext{
${ }^{3}$ For instance, the few early estimates of the IFR available around the time of data collection for this study (i.e., in March 2020) were based on non-US data, primarily from Asia (see the meta analysis by Meyerowitz-Katz and Merone, 2020), which may not be representative of the USA, given local differences in factors such as public health and healthcare access and quality.
}

reason, our information treatments entailed presentations of hypothetical scenarios to participants, as communicated to participants prior to the treatments (Step 2 below).

Participants in our survey experiment were assembled by the survey company Qualtrics, who was instructed to recruit a sample of 3000 survey respondents representative of the US general population in gender, age, income, education, race, and residential region. Due to oversampling by Qualtrics, our total sample size is $N=3133$. The advantage of using Qualtrics over less costly alternatives, such as Amazon Mechanical Turk or Turk Prime, is that Qualtrics continuously performs quality checks of their participants, including with regard to background characteristics and screens for professional survey takers, which are otherwise known to contaminate online panels (see, e.g., Chandler and Paolacci, 2017, and Sharpe Wessling et al., 2017). Participants received standard compensation for completing a Qualtrics survey. An additional benefit was that Qualtrics could offer rapid data collection, which was important given the information flow on COVID-19 that participants were exposed to outside of our study. Approximately $80 \%$ of the data were collected between March 24 and March 31, 2020.

The sequence of the study was as follows:

Step 1: Participants were asked questions about their gender, age, education, race, income, and region of residence, to ensure the sample met US national quotas for those characteristics.

Step 2: All participants received the following information about COVID-19:

Coronaviruses (CoV) are a large family of viruses that cause illness ranging from the common cold to more severe diseases.

Common signs of infection include respiratory symptoms, fever, cough, shortness of breath, and breathing difficulties. In more severe cases, infection can cause pneumonia, severe acute respiratory syndrome, kidney failure, and even death.

The new coronavirus (COVID-19) is still spreading globally, meaning that the risks to average Americans of catching the disease (currently or in a near future) are still uncertain, as are the risks of developing symptoms severe enough to cause deaths. In this study, we will present you with plausible estimates of these risks, based on recent knowledge of the virus and associated risks.

Public health risks caused by infectious diseases are often communicated by the Centers for Disease Control and Prevention (CDC). The CDC was established in 1946 and is the leading National Public Health Institute in the USA. It is 
a federal agency under the Department of Health and Human Services. CDC's goal is to protect public health and safety through the control and prevention of disease, injury, and disability.

Step 3: Participants were randomized into one of the eight information treatments.

If randomized into one the four treatments with CDC information only, participants saw the following statement:

The Centers for Disease Control and Prevention (CDC) has estimated that the probability of catching the coronavirus in the next 12 months is 25 [85] percent for the average American, meaning that 25 [85] out of 100 Americans are expected to catch the coronavirus.

Medical scientists have estimated that 1.5 [10] percent of Americans who catch the coronavirus will experience severe consequences leading to death, meaning that 15 [100] out of 1000 Americans who catch the virus are expected to die.

If instead randomized into one of the four treatments with both CDC and White House information, participants saw the following statement:

The Centers for Disease Control and Prevention (CDC) has estimated that the probability of catching the coronavirus in the next 12 months is 25 [85] percent for the average American. In other words, the CDC estimates that 25 [85] in 100 Americans will catch the virus.

The White House has indicated that the probability of catching the coronavirus in the next 12 months is lower, namely 10 [70] percent for the average American. In other words, the White House predicts that 10 [70] in 100 Americans will catch the virus.

Medical scientists have estimated that 1.5 [10] percent of Americans who catch the coronavirus will experience severe consequences leading to death. In other words, 15 [100] out of 1000 Americans who catch the virus are expected to die.

Two considerations were important to us when choosing the range of both the probability of infection and the IFR to include in the experimental design of our survey. First, we considered statistical efficiency: A reasonably large difference in the levels between treatments is needed to achieve a sufficiently precise estimate of the relevant treatment effect (in this case, the effect on an individual's willingness to get vaccinated). Second, we aimed to set the design levels in a plausible range for our survey participants at the time. That said, what might constitute a "plausible range" may be hard to determine, both because the risks of the virus are unknown due to limited testing and because the risks may change across time, as a result of changes to policies and individual responses to the virus.
As shown, if participants were treated with information about the probability of infection from the CDC only, then there was no discrepancy in the risk information presented to participants, and high probability of infection $=85 \%$, while low probability of infection $=25 \%$. As a reference point for the high probability of infection, we used the Imperial College COVID-19 Response Team's estimate from mid-March 2020, suggesting that more than $80 \%$ of the UK and US populations could be infected during the course of the pandemic, if individual mixing behavior remained largely unaffected by the pandemic (Ferguson et al., 2020). Further, as shown above, if participants were treated with information about the probability from both the CDC and the White House, the messages from these sources were inconsistent. In these treatments, the probabilities communicated by the CDC were complemented by lower probabilities from the White House (the White House's high probability $=70 \%$; the White House's low probability $=10 \%){ }^{4}$ Hence, while the probabilities communicated by the White House also varied between high and low, they were consistently lower (more optimistic) than the probabilities communicated by the CDC. The information treatments attributed statements of risk made by the CDC or White House officials that are not direct quotes but rather are paraphrased summaries designed to provide a clean contrast between the severity of the risk communicated by the two sources, capturing the observation that the White House generally communicates lower risk (CNN, 2020; MSN, 2020).

The above text excerpts from the survey also show that the source of the treatment information on the IFR of the disease was stated to be "medical experts" and high mortality $($ IFR $)=10 \%$, while low mortality $($ IFR $)=1.5 \%$. While the $10 \%$ level was higher than the contemporaneous central estimates in March 2020 (e.g., Shereen et al. (2020) estimated an IFR at around 3\%, across 109 countries, and Cascella et al. (2020) reported a mortality rate of $1-2 \%$, across multiple studies), we judged that this range would be viewed as plausible by most survey participants, especially in light of the wide variation in the early reports of COVID-

\footnotetext{
${ }^{4}$ We kept the disparity in risk communicated by the CDC and the White House constant (at 15 percentage points) across both high and low infection risk treatments. Viscusi (1997) shows that the disparity in the risks communicated by different information sources may affect trust in all information sources, such that a change in the disparity in percentage point probabilities across high and low infection risk treatments could affect trust in both the CDC and the White House.
} 
19 risks. ${ }^{5}$ As points of reference for this higher range, we considered the high estimates of the case fatality ratio (CFR) in March from Asia and Italy for individuals with underlying health conditions, which were close to $10 \%$ (Onder et al., 2020 and Jung et al., 2020). We also considered fatality ratios for previous coronaviruses. Common estimates of the CFR for SARS and MERS are 10\% and $34 \%$, respectively (e.g., CDC, 2020b, and WHO, 2020b). Much of the high initial uncertainty about the IFR in the USA has, however, been resolved since data collection for our survey concluded in March 2020. Central estimates of the IFR from studies that account for asymptomatic infections suggest that the IFR is likely below $1 \%$ (Meyerowitz-Katz and Merone, 2020).

Step 4: All participants were asked to indicate their beliefs about the probability that they and their children (if they had children) will catch COVID-19 (higher/lower/ about the same as the probability for the average American) and the probability that the average American will catch COVID-19. Similarly, they were asked about the conditional mortality risk (i.e., the IFR) for themselves and their children (higher/lower/about the same as the conditional mortality rate of the average American indicated to them in the survey).

Step 5: All participants were asked whether they would vaccinate for COVID-19. Before the vaccine question, they were given additional information on the risks and benefits of the vaccine. Because many people worry about vaccine side effects, and because those worries might be elevated when a vaccine is produced in a relatively short amount of time, we included information about the vaccine being approved by the Food and Drug Administration (FDA), following standard protocols. Specifically, participants were given the following information and question about whether they would take the vaccine:

Numerous pharmaceutical companies are working to develop a vaccine against the coronavirus. Before any vaccine can be provided to the public, the United States Food and Drug Administration (FDA) must approve its use. The FDA

\footnotetext{
${ }^{5}$ While the IFR in our study is high, our data imply that both the $1.5 \%$ and $10 \%$ IFR indicated in our survey were viewed as plausible by most study participants. After presenting respondents with our chosen design levels for the IFR for the average American (i.e., $1.5 \%$ or $10 \%$ ), participants were asked whether they believed their own risk was lower, about the same, or higher than the indicated level. In all treatments, $65-70 \%$ of participants stated that they either considered themselves to be at "about the same" or "higher" risk, and we find no statistically significant (or substantive) difference in those beliefs across respondents in the low- and highmortality treatments.
}

grants approval only if the vaccine is manufactured in compliance with all current regulations and medical scientists find that the vaccine is effective and has minimal side effects.

Suppose that the vaccine was approved for use by the FDA and was available today from your healthcare provider for free.

Also suppose that the vaccine is as effective as the flu vaccine in an average year, which is about 60 percent. In other words, 60 out of 100 people who are vaccinated would be protected from the coronavirus.

Would anyone in your family get the coronavirus vaccine under the conditions described above?

Participants were asked to indicate WOULD get vaccinated or WOULD NOT get vaccinated, for themselves. If they were parents of minors, they were also asked whether they would vaccinate their child, if they had one child, and whether they would vaccinate their youngest and oldest child, if they had multiple children. If they indicated one or more family members would not get vaccinated, they were presented with a series of follow-up questions designed to investigate the reasons for their choice. ${ }^{6}$

Step 6: Participants were asked about behavior undertaken to protect themselves from COVID-19 (hand washing, avoidance of crowds and public spaces, etc.), if they (or their children) had received a flu shot in the last 2 years, if they were vaccinated for measles, if they generally followed the recommended immunization schedule for children, and questions underlying the psychological scale for vaccinations (for measles and flu vaccines) developed by Betsch et al. (2018).

Step 7: Participants were asked about their information sources on COVID-19 (family, friends, conservative media, liberal media, family physician, President Trump, etc.), and their views about the trustworthiness of a variety of information sources.

\footnotetext{
${ }^{6}$ Note that we asked participants to suppose that a vaccine that was available today, although we expected participants to understand that a vaccine was in fact not yet available. An alternative would have been to ask about intentions to vaccinate at a future point in time, when a vaccine is more likely to be available. Our choice is based on control over the study environment. Participants may differ in their beliefs about when a vaccine will be available and how the risks of infection and death may evolve over the course of the outbreak-they might expect the pandemic to have concluded before a vaccine is available, herd immunity to be near, or that they personally will already have been infected. The recent polls that have measured COVID-19 vaccine hesitancy (see discussion in Sect. 4) vary in how they have dealt with the timing of the vaccine when asking about the willingness to vaccinate. Like our study, the poll by Pew Research Center (2020) asks about vaccine intentions if the vaccine was available today, while the polls by ABC news/Ipsos (2020) and LX/ Morning Consult (2020) ask about willingness to vaccinate when a vaccine becomes available without specifying when that might be.
} 
Step 8: Participants were asked about underlying health conditions that would put them at higher risk of severe consequences if they were to develop COVID-19 (e.g., respiratory disease, cardiovascular disease, obesity, diabetes, cancer, etc., see CDC, 2020c). They were also asked about risk factors for contracting the virus (being a healthcare worker, living in an urban area, etc.).

Step 9: Participants were asked about their religious beliefs, questions underlying the social and fiscal conservatism scale developed in Everett (2013), and their views about the currently implemented social distancing measures in the USA.

The full experimental survey is given in Supplemental online appendix.

Fifty-two percent of participants are female, and the mean age in our sample is 46 years. Fifty-seven percent of participants have a minimum of some college education. Twenty-five percent of participants fall into the low-income category ( $\$ 24,999$ per year or less), 55 percent into the medium-income category ( $\$ 25,000-\$ 99,999$ per year), and $20 \%$ into the high-income category $(\$ 100,000$ per year or more). About $37 \%$ of participants identify as Republican, $41 \%$ as Democrat, and $22 \%$ identify with neither political party. Nearly $37 \%$ of our participants live in a rural area, and $81 \%$ believe in God. Around $55 \%$ of our sample had the flu shot in the last 2 years, and participants were nearly evenly split among the low, middle, and high levels of trust in government agencies. Of adult participants in our sample, $82 \%$ have followed the recommended vaccination schedule, and of participants with children, $86 \%$ have followed the recommended vaccination schedule for their children. Supplemental online appendix includes a table with a more complete set of descriptive statistics, including the variables in the regression that generates (Fig. 4).

The randomization of participants into the different treatment groups resulted in relatively balanced sample sizes across all treatment groups, ranging from 363 to 413 participants in each group. (An overview of the treatment groups is provided in Supplemental online appendix.) Our primary analysis of the treatment effects relies on statistical analysis that tests the presence of these effects without controlling for other variables that may affect vaccine intentions. Our ability to identify the treatment effects by excluding other control variables in the statistical analysis relies on the assumption that the randomization of participants into treatments was successful in eliminating any meaningful differences in relevant covariates across treat- ments. If the randomization is unsuccessful, such that the value of covariates that are a priori expected to impact the outcome variable (e.g., general vaccine hesitancy) differ across treatment groups, then Athey and Imbens (2017) and Mutz et al. (2017) argue that it is appropriate to control for those covariates in the statistical analysis. Therefore, we also examined whether relevant covariates (those included in Fig. 4 that we expected not to be affected by the treatments) differ across treatments in meaningful ways. We followed Imbens and Rubin (2015) and identified "meaningful" differences by calculating normalized differences in mean values of covariates across pairs of treatments and designating an absolute value of the normalized difference as meaningful if it exceeds 0.25 in absolute value. For no covariate, in any pairwise comparison of treatments, did we find a value close to 0.25 (see Supplemental online appendix for details on the outcome of normalized differences in means across subject characteristics and attitudes across all treatments). We therefore concluded that the randomization in our experiment was successful, which in turn suggests that we should refrain from including covariates in our statistical analysis of treatment effects.

\section{Results}

\section{Prevalence of COVID-19 Vaccine Avoidance Across Risk, Mortality, and Mixed Information}

First, we examined whether the treatment information on the probability of infection affected participants' beliefs about the risk of the average American becoming infected in the next 12 months. Figure 1 shows histograms of risk perception across treatments.

While Fig. 1 shows substantial heterogeneity in the beliefs about the risk of infection of the average American, it is notable that the high/low probability of infection treatment induced variation in the perceived probability: The distribution is skewed to the right in the high-probability treatments and to the left in the low-probability treatments. Figure 1 also shows that participants to some extent gravitated toward stating a probability of 50 percent, which is in line with findings from previous research about stated probabilities for uncertain events (Bruine de Bruin et al., 2002).

Panel (a) of Fig. 2 shows the mean values of participants' beliefs about the risk of the average American becoming infected in the next 12 months across the eight 


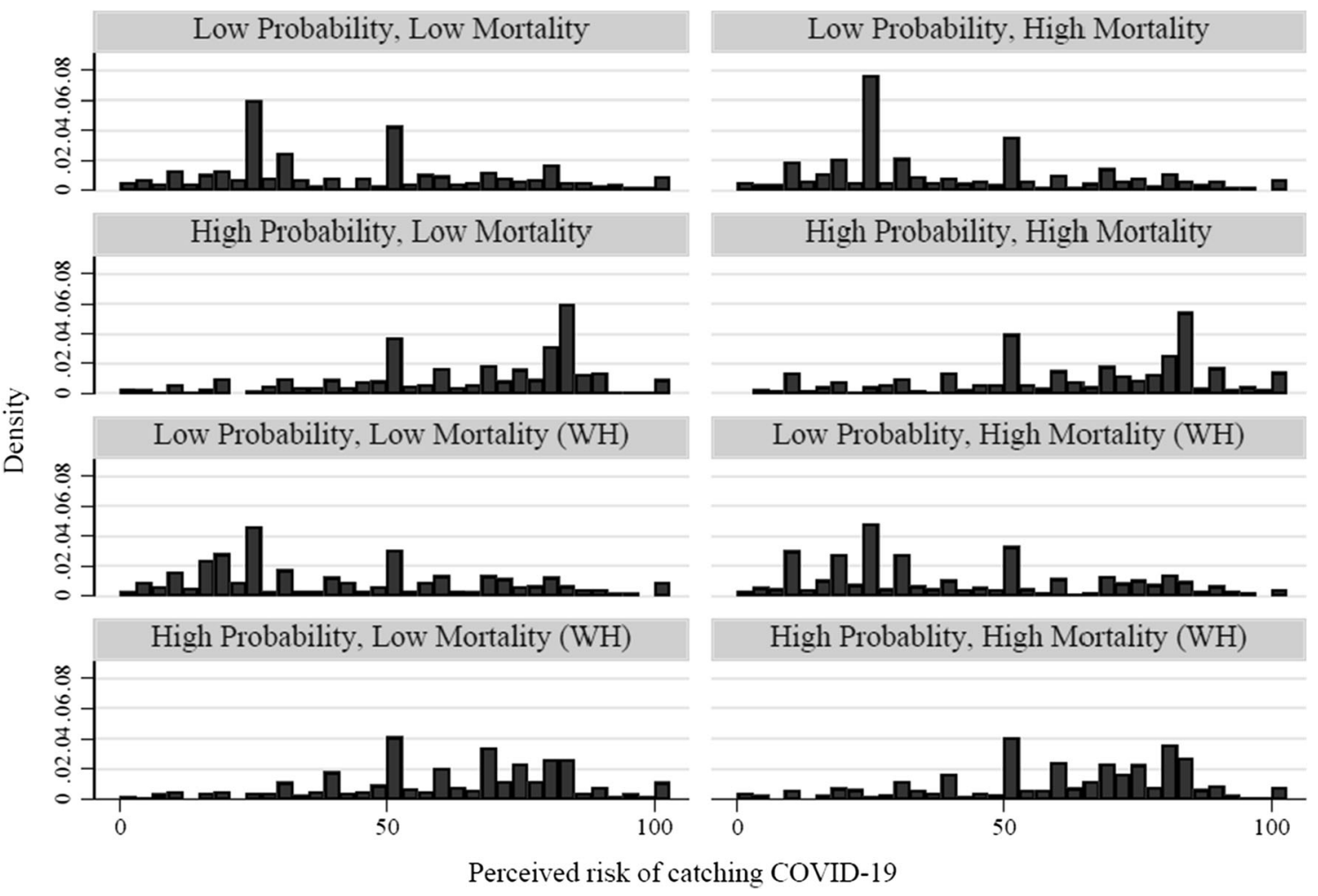

Fig. 1. Density distributions of perceived probability of infection across treatments

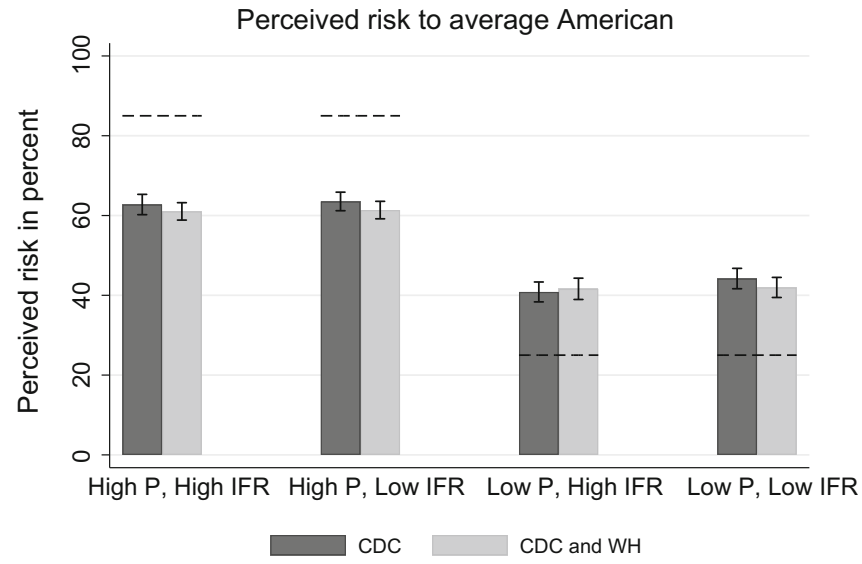

(a)

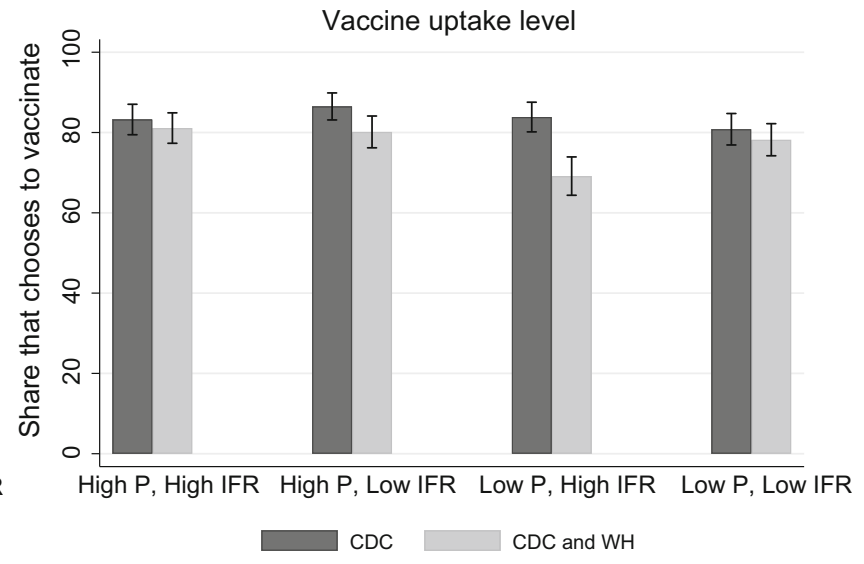

(b)

Fig. 2. Mean perceived risk of infection and vaccine uptake across treatments

treatment groups. It shows that the mean differences in risk perceptions across high- and low-probability treatments are large in magnitude (around 20 percentage points across all four comparisons of perceived risk in high- versus lowprobability treatments). Visually, panel (a) of Fig. 2 also suggests that participants' mean risk perceptions are somewhat lower in three out of four treatment comparisons when risk information is communicated by both the
CDC and the White House, compared to when communicated by the CDC only.

To examine treatment effects on perceived probability of infection, we used a zero-one inflated beta regression. Average marginal effects from the zero-one inflated beta regression are shown in Fig. 3.

As shown in Fig. 3, the results from the zero-one inflated beta regression imply that the information about low 


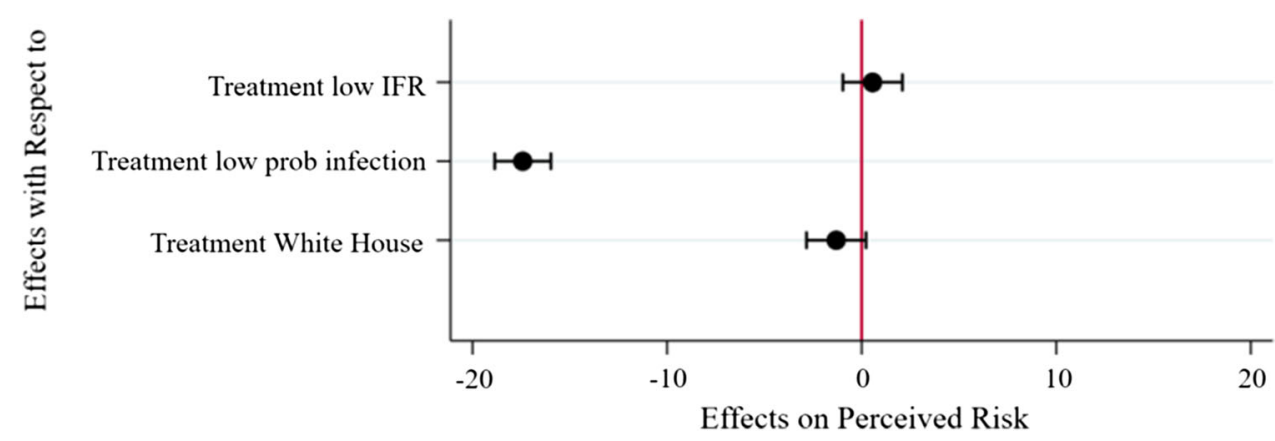

Fig. 3. The effects of treatments on perceived risk-marginal effects from a zero-one inflated beta regression

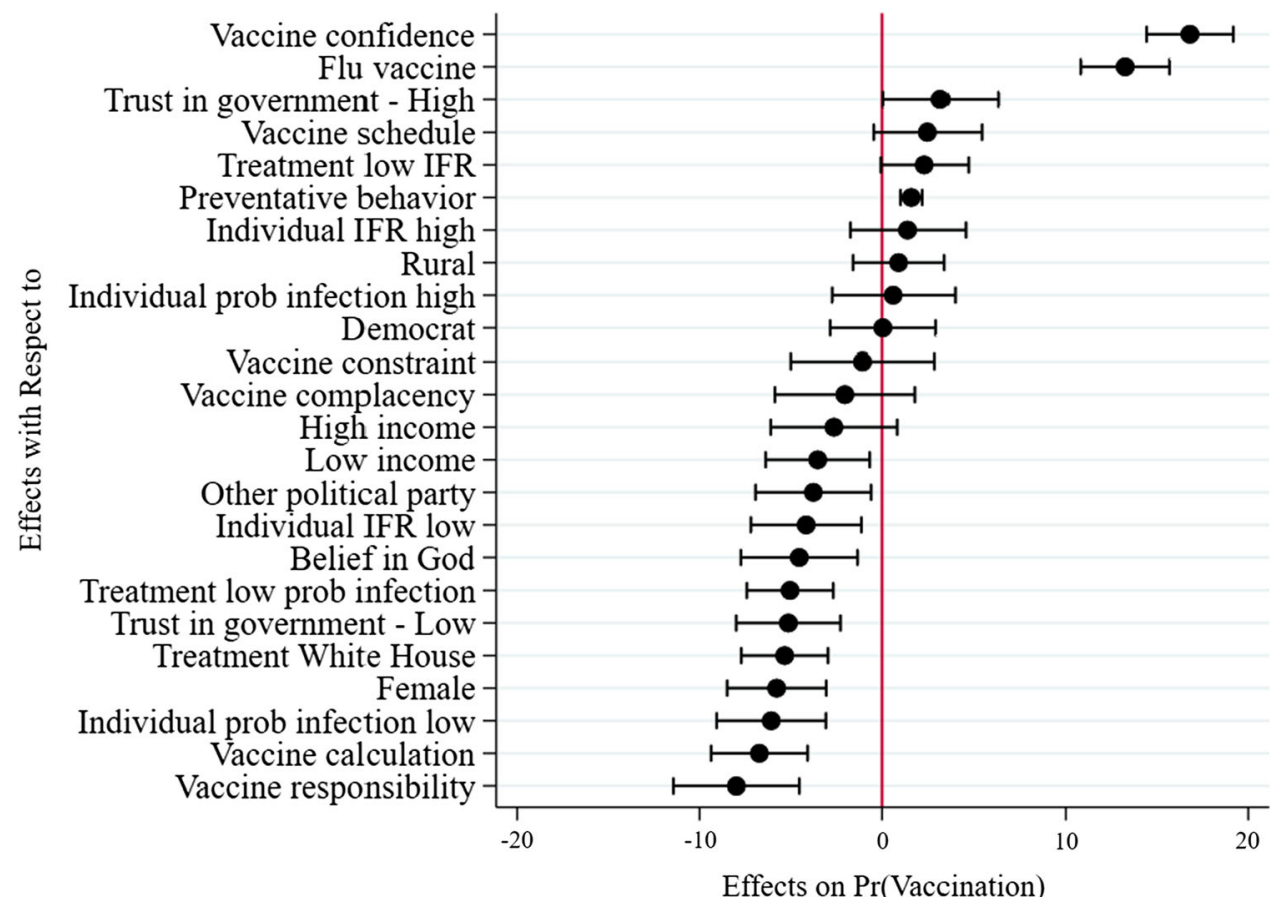

Fig. 4. Determinants of decision to vaccinate for COVID-19-average marginal effects from a probit regression

probability of infection reduces perceived probability of infection by 17.4 percentage points (adjusted $p=0.003$ ), which is consistent with the results displayed in Fig. 2a. ${ }^{7}$ As should be expected, the information on the IFR from COVID-19 does not affect the perceived probability of infection (its average marginal effect is close to zero; adjusted $p=1.000$ ), given the perceived infection risk only pertains to the probability of catching the disease, not its mortality rate conditional on infection. The results from the regression also suggest that we cannot detect an effect

\footnotetext{
${ }^{7}$ All results are adjusted for multiple hypothesis testing using the Bonferroni correction (Bonferroni, 1935) with a family-wise type I error rate of 0.05 . Adjusted $p$ values represent Bonferroni corrected $p$-values.
}

on perceived infection risk from the more optimistic risk information communicated by the White House (the average marginal effect is both small (1 percent) and not statistically significant; adjusted $p=0.282$ ).

Further, Fig. 1, panel (a) of Fig. 2, and Fig. 3 all show that while the treatments succeeded in affecting risk beliefs, participants were not basing their risk beliefs entirely on the information provided to them in the experiment. Specifically, the mean perceived risk to the average American in the four high-probability treatment groups in panel (a) of Fig. 2 range between 61 and 63.5\%, which is substantially lower than the probability of catching COVID-19 communicated by the high-probability treatment-recall that 
participants in the high-probability treatment were told either that the probability for the average American of catching COVID-19 in the next 12 months was $85 \%$ (if getting information from the CDC only-shown by the dotted lines at the 85 percent mark in panel (a), Fig. 2) or $85 \%$ according to the CDC and $70 \%$ according to the White House. Similarly, the mean perceived risk in the lowprobability treatment groups ranges from 40.8 to $44.2 \%$, which is substantially higher than the level of risk communicated by the low-probability treatments. Participants in these treatments were told either that the probability for the average American of catching COVID-19 in the next 12 months was $25 \%$ (if getting information from the CDC only-shown by the dotted lines at the 25 percent mark in panel (a), Fig. 2) or 25\% according to the CDC and $10 \%$ according to the White House. ${ }^{8}$

Next, we proceed to the focal point of our study and examine COVID-19 vaccine uptake across treatments. When we pool participants in all treatments, we find that $19.5 \%(n=612 / N=3133)$ of adults do not intend to vaccinate themselves and $19.7 \%(n=228 / n=1156)$ of those with children do not intend to vaccinate their children against COVID-19. Panel (b) of Fig. 2 shows that the share of people who intend to vaccinate is fairly large and consistent across treatment groups at around 80 percent or higher. The exception is the group with a low probability of infection and high IFR that received information from both the CDC and the White House, in which 69 percent stated an intention to get vaccinated.

Across treatment groups, we varied (1) the probability of infection for the average American, (2) the IFR of the average American, and (3) whether the probability of infection is communicated by the CDC and the White House (and the White House downplays the risk). We estimated a probit regression to examine the effects of these three factors on the intention to vaccinate. The results from the probit regression show that the vaccine uptake (i.e., the intention to vaccinate) decreases by 5 percent if the probability of being infected is low compared to high (adjusted $p=0.003)$ and decreases by 6 percent if inconsistent risk information is given by both the CDC and the White House, compared to by the CDC only (adjusted $p<0.001)$. Overall, these results suggest that inconsistent information about risks may affect vaccination intention, despite no discernible effect on perceived risk. We cannot

\footnotetext{
${ }^{8}$ The results reported in Fig. 2 (a) remain highly similar even if we remove subjects
} stating 50 percent (see figure included in Supplemental online appendix). statistically detect an effect of IFR on vaccine intentions (adjusted $p=0.498){ }^{9}$

Panel (b) of Fig. 2 visually displays that the share of people who intend to vaccinate generally increases as the probability of infection increases. It also shows that people's vaccination decisions are affected by the White House communicating lower probabilities than the CDC. The share of people who vaccinate is consistently lower in the treatments where the White House communicates lower probabilities of infection, compared to the CDC. In contrast, panel (b) of Fig. 2 shows that there is little consistency in the response to the information on IFR from COVID-19. If the probability of infection is low, and communicated by both the CDC and White House, it even seems like people are more likely to get vaccinated when the IFR is low compared to when it is high. This finding results from the particularly low vaccine uptake in the treatment group that received information, from both the CDC and the White House, with a low probability of infection and high IFR. ${ }^{10}$

Our finding of around 20 percent vaccine avoidance is consistent with results from polls conducted in the earlier stages of the pandemic. A survey by Pew Research Center (2020) found that $27 \%$ of US adults would not get a coronavirus vaccine if it was available today, a poll by $\mathrm{ABC}$ news/Ipsos (2020) suggested that $25 \%$ of US adults were

\footnotetext{
${ }^{9}$ The results from the probit regression are reported in Supplemental online appendix. It is the same model that generates the result in Fig. 4, but with the treatment variables only (i.e., only the top three variables in Fig. 3), given the isolation of the treatment effects relies on the randomization of participants across treatments. The observed treatment effects are very similar to those generated by the model in Fig. 4 . For space saving reasons, we therefore refrain from including a separate figure in the main text for the probit regression that has the treatment variables only.

${ }^{10}$ The particularly low vaccine uptake in the group that received the "low-probability (communicated by both the CDC and the White House) and high-mortality" treatment can be partially explained by our finding that the IFR for the average American (as communicated in our study) has little (if any) influence on participants' decision to vaccinate, as shown in Fig. 4. The probability of infection and the mixed messages from the White House and the CDC have larger effects on vaccine uptake (as suggested by the results in Fig. 4). Therefore, we should expect the vaccine uptake in this treatment to be lower than that in other treatments. Another factor that could contribute to the low vaccine uptake in this group is differences in participant characteristics between this group and the rest of the sample. As discussed in Sect. 2, we found no "meaningful" differences (Imbens and Rubin, 2015) in participant characteristics and attitudes across any treatment groups. However, there are small differences between this group and the rest of the sample (statistically significant at conventional levels, but uncorrected for multiple hypotheses testing). Specifically, the share of women, share of participants with low trust in government agencies, and share of participants ascribing to neither Democrats nor Republicans are all higher in this group than all other groups in the sample. These differences are in the direction that would suggest a lower than average vaccine uptake for this group, as suggested by our analysis of vaccine uptake for our sample as a whole.
} 
unlikely to get vaccinated if an effective coronavirus vaccine was developed, and a poll by LX/Morning Consult (2020) found that $9 \%$ of US adults would not get vaccinated if a vaccine became available, while another $15 \%$ did not know if they would get vaccinated.

Previous studies show that people find health risk information more believable if it is received from sources that share their values (Siegrist et al., 2000, 2001). For example, in a study of cancer cluster communication, Siegrist and colleagues (2001) found that people were more likely to believe clusters could occur randomly when they believed risk managers shared their values. Based on these findings, we hypothesized that individuals who identify as Republican or conservatives would respond the most to the White House information. To examine whether Democrats and Republicans differ in their response to the White House information, we re-estimated the zero-one inflated beta regression reported in Fig. 2 on a sub-sample of participants that identified as Republicans and Democrats only (i.e., excluding participants who did not identify with either political party). We also included two interaction terms: (1) Republican $\times$ the low-probability risk treatment and (2) Republican $\times$ the White House information treatment. However, we could not detect any differences in responses to the White House information across Republicans and Democrats; both groups appear to be just as responsive to the more optimistic White House information. Results are reported in Supplemental online appendix.

\section{Determinants of Vaccine Avoidance}

Next, we pooled participants from all treatments to examine a broad range of determinants of the vaccine decision. Figure 4 shows the average marginal effects generated by a probit regression on the intention to vaccinate for COVID-19.

We find that the most important determinant of the decision to take a COVID-19 vaccine is vaccine confidence. The average marginal effect for Vaccine confidence shows that people who are confident that vaccines are generally safe are $17 \%$ more likely to take the COVID-19 vaccine (adjusted $p<0.001) .{ }^{11}$ Further, we find that having taken the flu shot correlates with intentions of taking the COVID-19 vaccine. The marginal effect for Flu shot shows that

\footnotetext{
${ }^{11}$ The dummy variables Vaccine confidence, Vaccine complacency, Vaccine calculation, Vaccine collective responsibility, and Vaccine constraint represent the five key components of the vaccine hesitancy scale developed by Betsch et al. (2018).
}

people who took the flu shot in the last two years are $13 \%$ more likely to take the COVID-19 vaccine (adjusted $p<0.001)$. However, the estimated effect of whether a person followed the recommended vaccine schedule was not statistically significant (adjusted $p=1.000$ ).

The estimated effects of the treatment dummies in the probit regression are consistent with the results discussed in conjunction with panel (b) in Fig. 2. We do not find a statistically significant effect of the IFR (adjusted $p=1.000$ ), as shown by the marginal effect generated by the variable Treatment-Low IFR (which takes the value 1 if the treatment communicated low IFR, and 0 if it communicated high IFR). In contrast, we do find that if the probability of infection from COVID-19 is low, people are less likely to vaccinate (adjusted $p<0.001$ ). People are also less likely to vaccinate if the probability of catching the virus is communicated as 15 percentage points lower by the White House than the risk communicated by the CDC (adjusted $p<0.001$ ).

The variable Preventative Behavior represents the number (ranging from 0 to 12 ) of preventive measures (e.g., washing their hands more, becoming better informed, praying to stay resilient, and eating better) a participant takes to avoid getting infected by COVID-19. We find that people who self-protect more in other ways are also more likely to vaccinate for COVID-19 (adjusted $p<0.001$ ).

We do not find a statistically significant difference in vaccine intentions between rural and urban households (adjusted $p=1.000$ ). Also, we cannot detect effect beliefs about the individual mortality risk from COVID-19 deviating from that of the average American. Specifically, we do not find an effect of the risk being higher than that of the average American (adjusted $p=1.000$ ), or lower than that of the average American (adjusted $p=0.168$ ). Also, we do not find an effect on vaccine intentions from political identity. We do not find a difference in the probability of vaccinating for Democrats versus Republicans (adjusted $p=1.000$ ) or people who identify as neither Democrat nor Republican, as implied by Other political party (adjusted $p=0.480)$. We do not find an effect from people agreeing that everyday stress prevents them from getting vaccinated, as implied by the estimated marginal effect for Vaccine constraint (adjusted $p=1.000$ ). Similarly, we do not find an effect on the decision to vaccinate from people believing vaccines are unnecessary because vaccine preventable diseases are uncommon, as shown by the marginal effect for Vaccine complacency (adjusted $p=1.000$ ). 
Even though Fig. 4 shows that the 95 percent confidence intervals for Low income and Belief in God do not include zero, after adjusting their $p$-values for multiple hypothesis testing we find no statistically significant effect from these variables. Specifically, compared to our reference case (medium income), we find no statistically significant effect of low income (adjusted $p=0.360$ ) or high income (adjusted $p=1.000$ ). Further, we do not find that vaccine intentions are different for those who believe in God, compared to non-believers (adjusted $p=0.120$ ).

In contrast, we find that trust in government agencies might matter to people's vaccine decision. People with a low trust are $5 \%$ less likely to get vaccinated than those with a medium trust in government agencies (adjusted $p<0.001)$. This is consistent with previous findings on the positive correlation between government trust and vaccine uptake (Lee et al., 2016). However, the effect on vaccine uptake from trust might not be linear, since we do not find an effect on vaccine decisions from having a high trust in government agencies, compared to medium trust (adjusted $p=1.000) .^{12}$

We find that gender matters. Women are $6 \%$ less likely to vaccinate than men (adjusted $p<0.001$ ). Our data further suggest that individual risk perceptions may matter to the vaccine decision. Figure 4 shows that participants who believe their risk of infection is lower than that of the average American are 6\% less likely to get vaccinated (adjusted $p<0.001) .{ }^{13}$ On the other hand, the difference in vaccine intentions between participants who believe their risk is higher versus the same as that of the average American is much smaller, around $1 \%$, and is not statistically significant (adjusted $p=1.000$ ).

Finally, we find that people who indicate that they weigh benefits against costs for vaccines are almost $7 \%$ less likely to get vaccinated (adjusted $p<0.001$ ), as implied by the average marginal effect for Vaccine calculation. People

\footnotetext{
${ }^{12}$ While we measured trust in government agencies as a continuous variable, it is included in the regression as a categorical variable. The reason for this is to avoid problematic multicollinearity. There is high bivariate correlation between the continuous trust measure and the vaccine confidence and the flu shot variables, respectively. If the continuous trust variable is included in the regression, trust (misleadingly) appears to have no effect on vaccine uptake. However, if either vaccine confidence or the flu shot variable is removed from the regression, the continuous trust variable is highly statistically significant. Binning the trust variable into low, medium, and high trust eliminates the problematic multicollinearity.

${ }^{13}$ We do not know the causality for this relationship-while people might be less inclined to take a vaccine if they perceive the risk of the virus to be lower, it is also possible that people who are hesitant to vaccines are motivated to downplay the risk of the disease (for motivated risk beliefs, see, e.g., Kopetz and Woerner, 2021).
}

who agree that they do not need to get vaccinated if everyone else is vaccinated are $8 \%$ less likely to get a COVID-19 vaccine (adjusted $p<0.001$ ), as implied by the average marginal effect for Vaccine responsibility.

The effects reported in Fig. 4 are robust to a range of inclusions of other (non-significant) explanatory variables, such as college education, race, underlying health conditions that increases the risk of severe consequences if infected, or working in a high-risk profession (health care, teaching). They are also robust to the inclusion of either the compressed treatment variables (the top three variables in Fig. 4), all eight treatment group dummy variables, or no variables representing treatment effects. The outcome of these robustness checks is presented in Supplemental online appendix. Further, despite many variables measuring different aspects of vaccine hesitancy, the multicollinearity among the variables in the model underlying Fig. 4 is low, as implied by a variance inflation factor (VIF) of 1.27. Full cross tabs of risk beliefs and vaccine intentions are presented in Supplemental online appendix.

Next, we report the main reasons people state they choose not to vaccinate against COVID-19. All participants who stated that they did not intend to vaccinate themselves or their children were asked: "You indicated that one or more of your family members would not get vaccinated. Please mark the extent to which any of the below reasons mattered to your decision not to take the vaccine." The reasons are shown in Fig. 5, and participants could mark if these reasons did not matter at all, mattered some, or mattered a lot. Figure 5 shows the share of participants who stated the reason mattered some or a lot, of participants who indicated that they would not take the vaccine.

Figure 5 shows that at least $80 \%$ of those declining the vaccine stated vaccine novelty and worry about negative side effects as reasons for doing so. This is consistent with previous findings that people are particularly skeptical to new vaccines (Dubé et al., 2013). Other important reasons for declining the vaccine are doubts that the vaccine will in fact provide protection from catching the virus, general vaccine hesitancy (avoiding most vaccines), and the belief that COVID-19 is not severe enough to warrant vaccination.

\section{Discussion}

A vaccine for COVID-19 might be the best hope for ending the pandemic. Scientists have raced to develop vaccines, in 


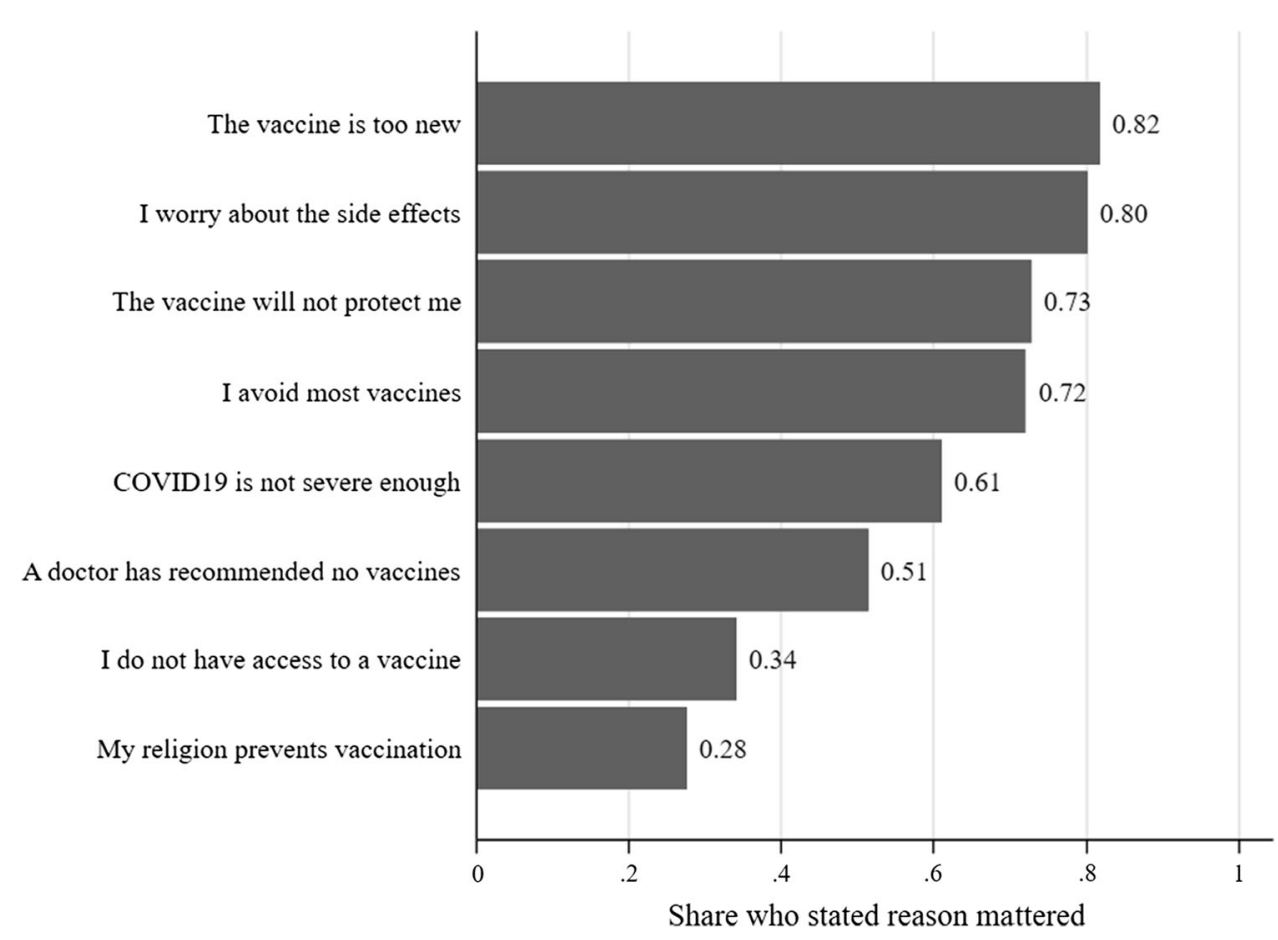

Fig. 5. Reasons for declining the COVID-19 vaccine

unprecedented joint efforts within the scientific community. However, as seen in the context of other diseases, in humans, agriculture, and wildlife, the challenge to extinguish the spread of a disease does not end with finding an effective vaccine. The implementation of the vaccine program is keenly important. In this study, we focus on the challenge posed to a vaccine program by vaccine hesitancy. What if large parts of the population decline the vaccine, once it is available? Vaccine hesitancy is well known for other types of vaccines and has increased in recent years (Dubé et al., 2013; Olive et al., 2018), for instance, causing recent outbreaks of measles (De Serres et al., 2013; Sarkar et al., 2019), a disease that was extinguished in the USA until recently.

We found that a substantial share (around 20 percent) of Americans intent to decline a COVID-19 vaccine that has been subjected to standard FDA vaccine safety protocols, if it was available today (i.e., end of March 2020, for the study participants). Even though we varied both the IFR and infection risk quite dramatically in our experimental survey, we found no consistent effect of the IFR on vaccine intentions, and the treatment effect of the infection risk is modest compared to the effects of other determinants of vaccine intentions. The infection risk treatment effect was estimated precisely enough to achieve statistical significance, which suggests that participants assimilated and trusted the risk information. But the small size of the effect suggests that they did not assign much weight to this factor. Our results suggest that avoidance of a COVID-19 vaccine is highly determined by general vaccine hesitancy, and the novelty of the vaccine and concerns about its safety are likely to decrease the uptake in the population even further. A prominent determinant of COVID-19 vaccine avoidance is whether a person had the flu shot in the last two years. This observation may be helpful in identifying people who are particularly reluctant to vaccinate, given the decision to take a flu shot is observable-healthcare providers, e.g., family physicians, have records (although perhaps incomplete) of who had flu shots.

The consequences of individuals' decisions whether (or not) to vaccinate on community outcomes have been mathematically illustrated (see, e.g., Fine and Clarkson, 1986). The key roles of perceived risk of the vaccine and the effectiveness of the vaccine in the decisions of individuals lead to a lower vaccine uptake than would be optimal for the community as a whole. Our elicited individual uptake rate of $80 \%$ (given the vaccine avoidance of 20\%) in combination with the effectiveness of a vaccine has implications on the potential proportion of the population immunized $\left(v_{e}\right)$. Policy makers might target a proportion of the population to be immunized with the deployment of a vaccine. However, the proportion immunized depends on 


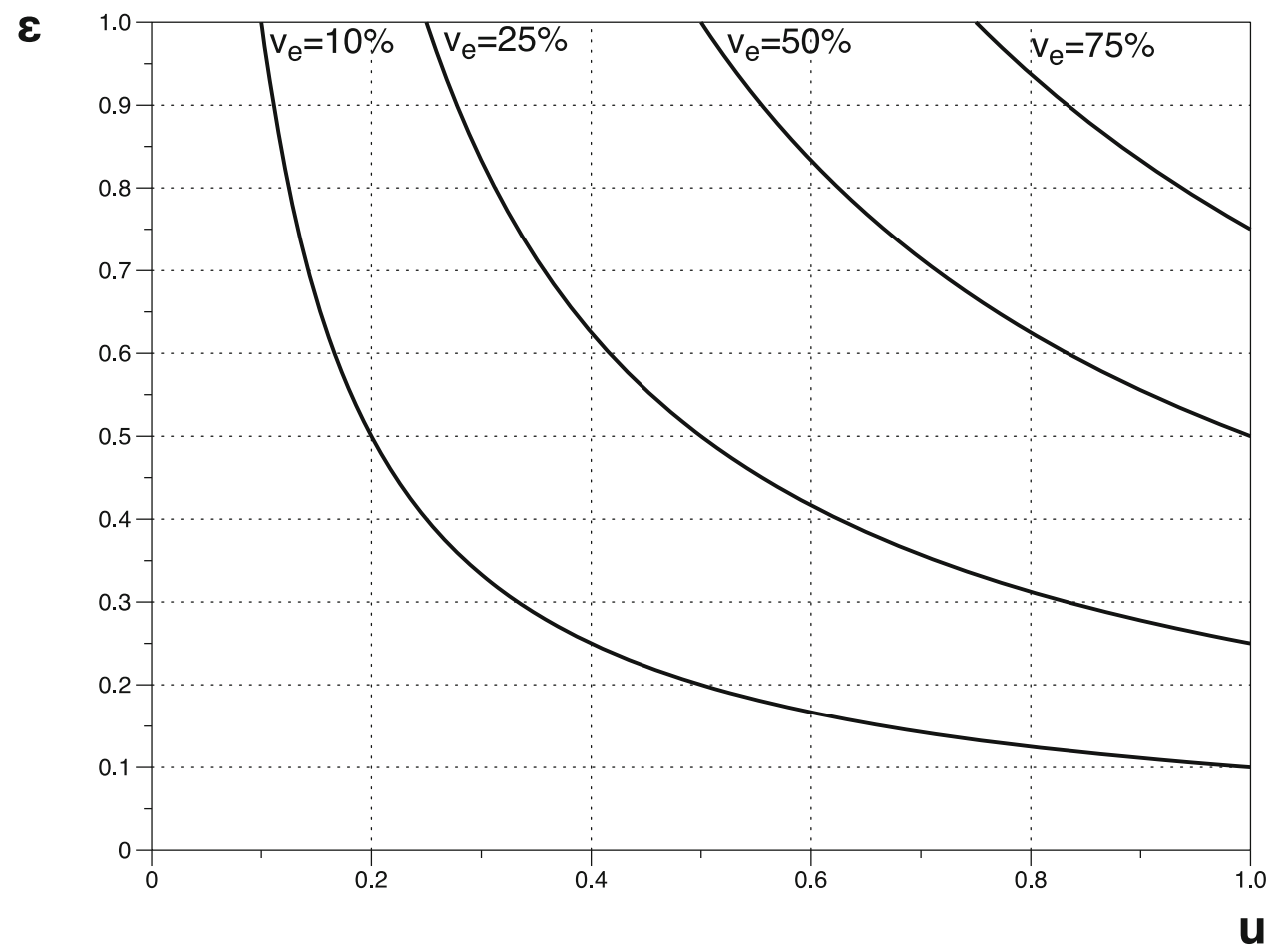

Fig. 6. Potential proportions of the population immunized

the effectiveness of the vaccine $(\varepsilon)$, the uptake rate $(u)$, and the proportion of the population given the opportunity to vaccinate $(v)$, where $v_{e}=\varepsilon u v$.

Figure 6 illustrates how the success of a vaccine program in immunizing alternative proportions of the population $\left(v_{e}\right)$ depends on the effectiveness of the vaccine $(\varepsilon)$ and the uptake rate $(u)$, if the entire population is given the opportunity to vaccinate $(v=1)$. For ranges of each parameter, contours for alternative levels of $v_{e}$ trace out the required combinations of $\varepsilon$ and $u$. For combinations of $\varepsilon$ and $h$ below and to the left of each contour, the proportion immunized is less than the contour proportion. Parameter combinations above and to the right of each contour result in proportions immunized higher than the contour proportion.

Let $u=0.80$ be our baseline value of vaccine uptake, as generated by our experimental study with $20 \%$ vaccine avoidance. For our baseline vaccine effectiveness of $\varepsilon=0.60$, since participants were told in the experimental survey that the COVID-19 vaccine would be $60 \%$ effective, based on this being the upper bound of effectiveness of the flu vaccine (another, but more rapidly mutating, RNA virus).

At these baseline values, Fig. 6 shows that if the entire population is given the opportunity to vaccinate, the proportion of the population immunized will be (at best) $48 \%$.
If, however, the effectiveness would be as high as $90 \%$ (as suggested by early vaccine trial results, see press release from Pfizer, November 9, 2020), then $72 \%$ of the population could be immunized, given the vaccine avoidance observed in our study. Note, however, that these results are generated under the rather optimistic assumption that all Americans are given the opportunity to take a COVID-19 vaccine.

Knowing about COVID-19 vaccine avoidance before a vaccine is available can help government agencies, healthcare workers, and other authorities mitigate the impact of vaccine avoidance. Such efforts may involve developing policies and a preparedness for the vaccine avoidance. It might also involve public information campaigns designed to increase confidence in the effectiveness and safety of the vaccine. Here, our results offer insights that may be helpful. First, our results suggest that an inconsistent risk message from government authorities may increase vaccine hesitancy. This result relates to findings that risk information in the news had direct effects on people's health behavior during the pandemic (Bursztyn et al., 2020). While the negative effect on vaccine uptake from mixed messaging is statistically significant, its importance to public health is uncertain, and may also depend on how different the 
messages are. In our study, we assumed the White House downplayed the risk by 15 percentage points and found that difference to cause a 6 percent reduction in vaccine uptake. Per our example above, this results in a reduction in the proportion immunized from 48 to $44 \%$ given a vaccine that is $60 \%$ effective. Second, we found that distrust in the government is higher among those who decline the vaccine. To address COVID-19 vaccine hesitancy, broader public health campaigns may therefore be less effective. Instead, efforts might focus on reaching out to healthcare providers (the most trusted source of vaccine safety information, see, e.g., Freed et al., 2011) and local authorities, including religious leaders. To identify effective strategies to reduce COVID-19 vaccine hesitancy, government agencies will likely benefit from the knowledge gathered during recent measles outbreaks-the COVID-19 vaccine decliners in our study share many attitudes with those generally avoiding vaccines (MacDonald, 2015). Policy makers may also consider regulations that require people to have COVID-19 vaccinations in order to attend schools and workplaces, similar in spirit to the bans of philosophical exemptions from vaccinations in the wake of the recent measles outbreaks (Kuehn, 2019).

Our study has several short comings. First, we only considered one level of vaccine effectiveness. (It would "protect" 60 percent of people who are vaccinated from the coronavirus.) The level of vaccine avoidance observed in our study could be unique to that effectiveness level. Further, while we intended our description of the vaccine effectiveness to signal that 60 percent of those vaccinated and exposed to the virus would not contract the virus, it is possible that participants interpreted the wording "protected from" to implicitly signal protection from severe consequences or death from COVID-19, which may also matter to the vaccine uptake. Second, COVID-19 risks are yet to be resolved, and the upper and lower bounds of risks communicated in our survey might be too low/high. In fact, much of the high initial uncertainty has been resolved since the time of our data collection. In the earlier stages of the pandemic, the prevalence of asymptomatic infections was largely unknown, such that perceived mortality rates were inferred primarily from the CFR (generally an overstatement of the IFR). For instance, around mid-2020, the CFR for the USA was estimated to be 5.1\% (Johns Hopkins University and Medicine (2020); Roser et al. (2020), which is lower than our upper bound of the conditional mortality rate $(10 \%)$ and higher than our lower bound (1.5\%). However, studies that account for asymptomatic infections suggest that the IFR is likely below $1 \%$ (Meyerowitz-Katz and Merone, 2020). Our results suggest that the fatality ratio for the average American, within the range explored in our study, might not matter much to vaccine decisions. ${ }^{14}$ However, future research might examine whether public knowledge of an IFR that is lower than the lower bound of our applied range might generate a different level of vaccine avoidance. We would expect that, all else equal, intentions to get a vaccine would be lower if the infection fatality ratio is known to be lower. Our results offer some indication in this direction-we found that participants who believe their individual IFR is lower than that of the average American (as communicated in our study) are less willing to vaccinate. More generally, the evolving knowledge of risks associated with COVID-19 suggests repeated studies or polls measuring vaccine avoidance might be beneficial.

We also encourage future research to examine the relationship between vaccine avoidance and costs. Participants in our study were asked to consider a costless COVID-19 vaccine. Getting a vaccine will be associated with some cost, whether it be a time, financial or inconvenience cost, such that the vaccine avoidance in our study is likely on the lower end. A study that measures the cost people are willing to bear for a COVID-19 vaccine may be helped by the literature examining willingness to pay for health risk reductions, see, e.g., Sloan et al. (1998), Smith and Desvousges (1987), Viscusi and Evans (1990), Viscusi and Aldy (2003), Edwards (2008), Hammitt and Haninger (2010), Alberini and Ščasný (2013), Finkelstein et al. (2013), and Gerking et al. (2017). Related, Serra-Garcia and Szech (2020) find that costs to COVID-19 antibody tests have substantial negative effects on people's willingness to get tested.

In the USA, conflicting risk messages about COVID-19 are regularly communicated to the public, by the media and public authorities, and have been shown to affect other types of health behavior related to COVID-19 (see, e.g., Bursztyn et al., 2020; Simonov et al. 2020). We encourage future research to further explore the mechanisms by which conflicting COVID-19 risk information affect vaccination decisions, see, e.g., Viscusi (1997), Viscusi et al. (1999), and Fox et al. (2002). Finally, other studies show that some preventive behaviors during the current pandemic are motivated by prosocial attitudes (Campos-Mercade et al., 2020; Jordan et al., 2020; Thunström et al., 2020). Future

\footnotetext{
${ }^{14} \mathrm{Also}$, the intended vaccine uptake for the treatments with low IFR only $(n=1,597)$ is $81 \%$, i.e., about the same as the average vaccine uptake for the study as a whole.
} 
studies might similarly examine whether prosocial messaging might increase a COVID-19 vaccine uptake.

\section{FUNDING}

We thank the College of Business Excellence Fund at the University of Wyoming.

\section{CODE AVAILABILITY}

All data and code are available upon request.

\section{Declarations}

\section{CONFLICT OF INTEREST The authors declare no} competing interests.

\section{REFERENCES}

ABC news/Ipsos poll (2020), Topline and Methodology, https:// www.scribd.com/document/460461528/ABC-News-Ipsos-PollMay-7-2020, Retrieved June 222020.

Aguas, R., Corder, R. M., King, J. G., Goncalves, G., Ferreira, M. U., and Gomes, M. G. M. (2020). Herd immunity thresholds for SARS-CoV-2 estimated from unfolding epidemics. medRxiv.

Alberini A, Ščasný M (2013) Exploring heterogeneity in the value of a statistical life: Cause of death v. risk perceptions. Ecological Economics 94:143-155

ASTHO. Communicating Effectively About Vaccines: Summary of a Survey of U.S. Parents and Guardians. Arlington, VA: Association of State and Territorial Health Officials, 2010.

Athey S, Imbens GW (2017) The econometrics of randomized experiments. In: Handbook of Economic Field Experiments, Banerjee AV, Duflo E (editors), Amsterdam, Netherlands: North-Holland, pp 73-140

Betsch, C., Schmid, P., Heinemeier, D., Korn, L., Holtmann, C., and Böhm, R. (2018). Beyond confidence: Development of a measure assessing the $5 \mathrm{C}$ psychological antecedents of vaccination. PLoS One, 13(12).

Binder AR, Hillback ED, Brossard D (2016) Conflict or caveats? Effects of media portrayals of scientific uncertainty on audience perceptions of new technologies Risk Analysis 36(4):831-846

Bonferroni CE (1935) Il calcolo delle assicurazioni su gruppi di teste, Rome: Tipografia del Senato

Breakwell GM (2000) Risk communication: factors affecting impact. British Medical Bulletin 56(1):110-120

Brewer NT, Chapman GB, Gibbons FX, Gerrard M, McCaul KD, Weinstein ND (2007) Meta-analysis of the relationship between risk perception and health behavior: the example of vaccination. Health Psychology 26(2):136

Bruine de Bruin W, Fischbeck PS, Stiber NA, Fischhoff B (2002) What number is "fifty-fifty"?: Redistributing excessive $50 \%$ responses in elicited probabilities Risk Analysis: an International Journal 22(4):713-723
Bursztyn, L. Rao, A., Roth, C. and Yanagizawa-Drott, D. (2020) Misinformation during a pandemic, Becker Friedman Institute Working paper 2020-44, https://bfi.uchicago.edu/wp-content/ uploads/BFI_WP_202044.pdf. Retrieved May 1.

Callaway E (2020) The race for coronavirus vaccines: a graphical guide-Eight ways in which scientists hope to provide immunity to SARS-CoV-2. Nature 580:576-577. https://doi.org/ 10.1038/d41586-020-01221-y

Calman K, Curtis S (2010) Risk communication and public health, : Oxford University Press

Cameron TA (2005) Updating subjective risks in the presence of conflicting information: an application to climate change. Journal of Risk and Uncertainty 30:63-97

Campos-Mercade , Meier A, Schneider F, Wengström E (2020) Prosociality predicts health behaviors during the COVID-19 pandemic. University of Zurich, Department of Economics, Working Paper, (346)

Carpenter DM, Elstad EA, Blalock SJ, DeVellis RF (2014) Conflicting medication information: prevalence, sources, and relationship to medication adherence. Journal of Health Communication 19(1):67-81

Cascella, M., Rajnik, M., Cuomo, A., Dulebohn, S. C., and Di Napoli, R. (2020). Features, evaluation and treatment coronavirus (COVID-19). In Statpearls [internet]. StatPearls Publishing.

Centers for Disease Control and Prevention (CDC) https://www. cdc.gov/flu/about/season/flu-season-2017-2018.htm, Retrieved April 20, 2020a.

Centers for Disease Control and Prevention (CDC), Frequently asked questions about SARS, https://www.cdc.gov/sars/about/fa q.pdf, Retrieved September 2, 2020b.

Centers for Disease Control and Prevention (CDC), https://ww w.cdc.gov/coronavirus/2019-ncov/downloads/COVID19-WhatYou-Can-Do-High-Risk.pdf, Retrieved April 4, 2020c.

Chandler J, Paolacci G (2017) Lie for a dime: When most prescreening responses are honest but most study participants are impostors. Social Psychological and Personality Science 8(5):500508

CNN, https://www.cnn.com/2020/04/07/politics/white-house-pre ss-secretary-coronavirus/index.html. Retrieved April 202020.

Cross ML, Buddle BM, Aldwell FE (2007) The potential of oral vaccines for disease control in wildlife species. The Veterinary Journal 174:472-480

De Serres G, Markowski F, Toth E, Landry M, Auger D, Mercier M, Bélanger P, Turmel B, Arruda H, Boulianne N, Ward BJ (2013) Largest measles epidemic in North America in a decade-Quebec, Canada, 2011: contribution of susceptibility, serendipity, and superspreading events. The Journal of Infectious Diseases 207(6):990-998

Dubé E, Laberge C, Guay M, Bramadat P, Roy R, Bettinger JA (2013) Vaccine hesitancy: an overview. Human Vaccines \& Immunotherapeutics 9(8):1763-1773

Edwards RD (2008) Health risk and portfolio choice. Journal of Business \& Economic Statistics 26(4):472-485

Everett, J. A. (2013). The 12 item social and economic conservatism scale (SECS). PLOS ONE, 8(12).

Ferguson, N. M., Laydon, D., Nedjati-Gilani, G., Imai, N., Ainslie, K., Baguelin, M., Bhatia, S., Boonyasiri, A., Cucunubá, Z., Cuomo-Dannenburg, G., Dighe, A., Dorigatti, I., Fu, H., Gaythorpe, K., Green, W., Hamlet, A., Hinsley, W., Okell, L. C., van Elsland, S., Thompson, H., Verity, R., Volz, E., Wang, H., Wang, 
Y., Walker, P. G. T., Walters, C., Winskill, P. Whittaker, C., Donnelly, A., Riley, S., Ghani, A. C. (2020) Report 9: Impact of non-pharmaceutical interventions (NPIs) to reduce COVID19 mortality and healthcare demand. Imperial College Response Team, http://hdl.handle.net/10044/1/77482.

Fine PEM, Clarkson JA (1986) Individual versus public priorities in the determination of optimal vaccination policies. American Journal of Epidemiology 124(6):1012-1020

Fine P, Eames K, Heymann DL (2011) "Herd immunity": a rough guide. Clinical Infectious Diseases 52(7):911-916

Finkelstein, A., Luttmer, E. F., and Notowidigdo, M. J. (2013). What good is wealth without health? The effect of health on the marginal utility of consumption. Journal of the European Economic Association, 11(suppl_1), 221-258.

Fontanet A, Cauchemez S (2020) COVID-19 herd immunity: where are we? Nature Reviews Immunology 20(10):583-584

Fox JA, Hayes DJ, Shogren JF (2002) Consumer preferences for food irradiation: how favorable and unfavorable descriptions affect preferences for irradiated pork in experimental auctions. Journal of Risk and Uncertainty 24(1):75-95

Freed GL, Clark SJ, Butchart AT, Singer DC, Davis MM (2011) Sources and perceived credibility of vaccine-safety information for parents. Pediatrics 127(Supplement 1):S107-S112

Frewer LJ, Howard C, Hedderley D, Shepherd R (1996) What determines trust in information about food-related risks? Underlying Psychological Constructs Risk Analysis 16(4):473486

Gerking S, Adamowicz W, Dickie M, Veronesi M (2017) Baseline risk and marginal willingness to pay for health risk reduction. Journal of Risk and Uncertainty 55(2-3):177-202

Hämeen-Anttila K, Nordeng H, Kokki E, Jyrkkä J, Lupattelli A, Vainio K, Enlund H (2014) Multiple information sources and consequences of conflicting information about medicine use during pregnancy: a multinational Internet-based survey. Journal of Medical Internet Research 16(2):e60. https://doi.org/ 10.2196/jmir.2939

Hammitt JK, Haninger K (2010) Valuing fatal risks to children and adults: Effects of disease, latency, and risk aversion. Journal of Risk and Uncertainty 40(1):57-83

Hoehn JP, Randall A (2002) The effect of resource quality information on resource injury perceptions and contingent values. Resource and Energy Economics 24(1-2):13-31

Imbens GW, Rubin DB (2015) Causal inference in statistics, social, and biomedical sciences, : Cambridge University Press

Johns Hopkins University and Medicine (2020), Mortality Analyses, https://coronavirus.jhu.edu/data/mortality, Retrieved June 262020.

Jordan, J., Yoeli, E., and Rand, D. G. (2020, April 3). Don't get it or don't spread it? Comparing self-interested versus prosocially framed COVID-19 prevention messaging. Doi: https://doi.org/ 10.31234/osf.io/yuq7x

Jung SM, Akhmetzhanov AR, Hayashi K, Linton NM, Yang Y, Yuan B, Kobayashi T, Kinoshita R, Nishiura H (2020) Real-time estimation of the risk of death from novel coronavirus (COVID19) infection: inference using exported cases. Journal of Clinical Medicine 9(2):523

Keeling MJ, Rohani P (2008) Modeling infectious diseases in humans and animals, Princeton, NJ: Princeton University Press

Kelly DL, Letson D, Nelson F, Nolan DS, Solís D (2012) Evolution of subjective hurricane risk perceptions: A Bayesian approach. Journal of Economic Behavior \& Organization 81(2):644-663
Kopetz C, Woerner JI (2021) People Downplay Health Risks to Fulfill Their Goals: A Motivational Framework for Guiding Behavioral Policy. Policy Insights from the Behavioral and Brain Sciences 8(1):92-100

Kuehn B (2019) Measles Vaccine Exemptions. JAMA 321(14):134. https://doi.org/10.1001/jama.2019.3063

Larson HJ (2018) The state of vaccine confidence. The Lancet 392(10161):2244-2246

Le, T.T., Andreadakis, Z., Kumar, A., Gómez Román, R., Tollefsen, S., Saville, M., and Mayhew, S. (2020) The COVID-19 vaccine development landscape. Nature Reviews Drug Discovery. Retrieved April 11. https://doi.org/10.1038/d41573-020-000735.

Leask J (2011) Target the fence-sitters. Nature 473(7348):443-445

Lee C, Whetten K, Omer S, Pan W, Salmon D (2016) Hurdles to herd immunity: Distrust of government and vaccine refusal in the US, 2002-2003. Vaccine 34(34):3972-3978

Liu, Y., Gayle, A. A., Wilder-Smith, A., and Rocklöv, J. (2020) The reproductive number of COVID-19 is higher compared to SARS coronavirus. Journal of Travel Medicine, 27(2). https://doi.org/ $10.1093 /$ jtm/taaa021

LX/Morning Consult (2020), National Tracking Poll \#200395: March 24-25, 2020. https://www.nbcwashington.com/wp-content/uploads/ 2019/09/LX_COVID_Vaccine_Poll_crosstabs.pdf. Retrieved June 22 2020.

MacDonald NE (2015) Vaccine hesitancy: Definition, scope and determinants. Vaccine 33(34):4161-4164

Magat, W. A. and Viscusi, W. K. (1992). Informational approaches to regulation (Vol. 19). MIT press.

Meyerowitz-Katz, G., and Merone, L. (2020). A systematic review and meta-analysis of published research data on COVID-19 infection-fatality rates. International Journal of Infectious Diseases, 101, 138-148. Mochiri, N. (2020) Coronavirus seems to mutate much slower than seasonal flu. https://www.livescience.c om/coronavirus-mutation-rate.html. Retrieved April 282020.

Mutz DC, Pemantle R, Pham P (2017) The perils of balance testing in experimental design: messy analyses of clean data. The American Statistician 73(1):32-42

MSN News (2020) https://www.msn.com/en-us/news/politics/anal ysis-the-white-house-continues-to-downplay-the-coronavirus-th reat-to-much-of-the-country/ar-BB12evZY. Retrieved April 20.

Olive, J. K., Hotez, P. J., Damania, A., and Nolan, M. S. (2018). The state of the antivaccine movement in the United States: A focused examination of nonmedical exemptions in states and counties. PLoS Medicine, 15(6).

Onder G, Rezza G, Brusaferro S (2020) Case-fatality rate and characteristics of patients dying in relation to COVID-19 in Italy. JAMA 323(18):1775-1776

Pew Research Center (2020) Most Americans expect a COVID-19 vaccine within a year; $72 \%$ say they would get vaccinated, $h$ ttps://www.pewresearch.org/fact-tank/2020/05/21/most-america ns-expect-a-covid-19-vaccine-within-a-year-72-say-they-wouldget-vaccinated/. Retrieved June 222020.

Pfizer (2020), Pfizer and Biontech announce vaccine candidate against COVID-19 achieved success in first interim analysis from phase 3 study (press release), https://investors.pfizer.com/invest or-news/press-release-details/2020/Pfizer-and-BioNTech-AnnounceVaccine-Candidate-Against-COVID-19-Achieved-Success-in-FirstInterim-Analysis-from-Phase-3-Study/default.aspx, Retrieved November 92020. 
Pushkarskaya H, Smithson M, Joseph JE, Corbly C, Levy I (2015) Neural correlates of decision-making under ambiguity and conflict. Frontiers in Behavioral Neuroscience 9:325. https:// doi.org/10.3389/fnbeh.2015.00325

Rémy, V., Largeron, N., Quilici, S., and Carroll, S. (2015). The economic value of vaccination: why prevention is wealth. Journal of Market Access \& Health Policy, 3(1), https://d oi.org/10.3402/jmahp.v3.29284.

Rodgers W (1999) The influences of conflicting information on novices and loan officers' actions. Journal of Economic Psychology 20(2):123-145

Roser, M., Ritchie, H., Ortiz-Ospina, E. and Hasell, J. (2020) Coronavirus Pandemic (COVID-19). Published online at OurWorldInData.org. https://ourworldindata.org/coronavirus. Retrieved April 282020.

Roth JA (2011) Veterinary vaccines and their importance to animal health and public health. Procedia in Vaccinology. 5:127136

Rousu, M. C., and Shogren, J. F. (2006). Valuing conflicting public information about a new technology: the case of irradiated foods. Journal of Agricultural and Resource Economics, 642652.

Sanche S., Lin, Y.T., Xu, C., Romero-Severson, E., Hengartner, N., and $\mathrm{Ke}, \mathrm{R}$. (2020). High contagiousness and rapid spread of severe acute respiratory syndrome coronavirus 2. Emerging Infectious Disease. 2020 Jul [11 April 2020]. https://doi.org/10. 3201/eid2607.200282

Sarkar S, Zlojutro A, Khan K, Gardner L (2019) Measles resurgence in the USA: how international travel compounds vaccine resistance. The Lancet Infectious Diseases 19(7):684-686

Sell TK (2017) When the next disease strikes: How to communicate (and how not to). Health Security 15(1):28-30

Serra-Garcia, M., and Szech, N. (2020). Demand for COVID-19 Antibody Testing, and Why It Should Be Free, CESifo Working Paper No. 8340, https://ssrn.com/abstract $=3623675$

Sharpe Wessling K, Huber J, Netzer O (2017) MTurk character misrepresentation: Assessment and solutions. Journal of Consumer Research 44(1):211-230

Shereen, M. A., Khan, S., Kazmi, A., Bashir, N., and Siddique, R. (2020). COVID-19 infection: origin, transmission, and characteristics of human coronaviruses. Journal of Advanced Research.

Siegrist M, Cvetkovich G, Roth C (2000) Salient value similarity, social trust, and risk=benefit perception. Risk Analysis 20(3):353-362
Siegrist M, Cvetkovich GT, Gutscher H (2001) Shared values, social trust, and the perception of geographic cancer clusters. Risk Analysis 21(6):1047-1053

Simonov, A., Sacher, S. K., Dubé, J. P. H., and Biswas, S. (2020). The persuasive effect of fox news: non-compliance with social distancing during the covid-19 pandemic (No. w27237). National Bureau of Economic Research.

Sloan FA, Viscusi WK, Chesson HW, Conover CJ, WhettenGoldstein K (1998) Alternative approaches to valuing intangible health losses: the evidence for multiple sclerosis. Journal of Health Economics 17(4):475-497

Smith VK, Desvousges WH (1987) An empirical analysis of the economic value of risk changes. Journal of Political Economy 95(1):89-114

Sy KTL, White LF, Nichols BE (2020) Population density and basic reproductive number of COVID-19 across United States counties. MedRxiv . https://doi.org/10.1101/2020.06.12.20130021

Thunström L, Newbold S, Finnoff D, Ashworth M, Shogren JF (2020) The benefits and costs of social distancing to flatten the curve for Covid-19. Journal of Benefit-Cost Analysis . https://doi.org/10.1017/ bca.2020.12

Viscusi WK (1997) Alarmist decisions with divergent risk information. The Economic Journal 107(445):1657-1670

Viscusi WK, Aldy JE (2003) The value of a statistical life: a critical review of market estimates throughout the world. Journal of Risk and Uncertainty 27(1):5-76

Viscusi, W. K., and Evans, W. N. (1990). Utility functions that depend on health status: estimates and economic implications. The American Economic Review, 353-374.

Viscusi WK, Magat WA (1992) Bayesian decisions with ambiguous belief aversion. Journal of Risk and Uncertainty 5(4):371-387

Viscusi WK, Magat WA, Huber J (1999) Smoking status and public responses to ambiguous scientific risk evidence. Southern Economic Journal 66(2):250-270

World Health Organization (WHO), https://www.who.int/newsroom/feature-stories/ten-threats-to-global-health-in-2019, Retrieved April 21 2020a.

World Health Organization (WHO), Middle East respiratory syndrome coronavirus (MERS-CoV). https://www.who.int/eme rgencies/mers-cov/en. Retrieved August 26 2020b. 